\title{
Treatment of tic douloureux with a new anticonvulsant (clonazepam)
}

\author{
JAIME E. COURT AND CARLOS S. KASE \\ From the Neurology Department, Catholic University, Santiago, Chile
}

SYNOPSIS Twenty-five patients affected by 30 episodes of tic douloureux were treated with a new anticonvulsant, clonazepam. In $40 \%$ there was complete control of the neuralgia and an additional $23.3 \%$ were significantly helped by the drug. Sixteen patients had previously been resistant to carbamazepine, and eight of them were completely and one partially relieved by clonazepam. The side-effects, somnolence and unsteadiness of gait, were present to some extent in 80 and $88 \%$ of the cases respectively, being severe in about half of them. It is concluded that clonazepam can be considered as a second choice for the treatment of this condition, after proven failure with carbamazepine. Possible mechanisms of action are discussed.

Tic douloureux is a condition characterized by brief paroxysms of extremely severe pain restricted to the distribution of one or more cutaneous branches of the trigeminal nerve. These often recur several times a day and are usually triggered by tactile or painful stimulation of the skin or mucosa innervated by branches of the trigeminal nerve. The episodes of neuralgia usually last for months or years, then subsiding for variable periods of time, to recur again with comparable severity. It is a disease of the adult, and its aetiology is unknown.

The first successful drug for its treatment was diphenylhydantoin (phenytoin, Dilantin, Epanutin) introduced by Braham and Saiz in 1960. In different series, it was reported that from $54 \%$ to $70 \%$ of patients were improved completely or significantly by this drug (Braham and Saiz, 1960; Blom, 1962, 1963). Because of its common side-effects (ataxia, dysarthria, nystagmus) and a significant incidence of recurrence of the neuralgia despite its continuous use, a new drug, carbamazepine (Tegretol), was introduced by Blom in 1962. Its reported effectiveness has been around 90\% (Blom, 1962), with most patients responding after 24 hours of treatment. It has been suggested that its effect on trigeminal Address for correspondence: Dr Carlos S. Kase, Universidad Catolica de Chile, Escuela de Medicina, Departamento de Anatomia Patologica, Casilla 114-D, Santiago, Chile.

(Accepted 23 October 1975.) neuralgia could be mediated by a depression of synaptic transmission on polysynaptic circuits of the trigeminal nuclei of the brain-stem, an effect demonstrated experimentally in the anaesthetized cat by Fromm and Killian (1967). The same action on the trigeminal nuclei of the cat had been previously demonstrated for diphenylhydantoin by Fromm and Landgren (1963).

During the last five years, there have been several reports on the efficacy of a new drug on epilepsy, clonazepam (Rivotril, Roche Laboratories) (Gastaut, 1970; Bonduelle and Sallou, 1973). This drug belongs to the group of the benzodiazepines. It is the 5-(2-chlorophenyl)1,3-dihidro-7-nitro-2 $\mathrm{H}-1,4$ benzodiazepine-2 ona. It is a modification of nitrazepam (Mogadon) by the addition of a chloride in the phenyl group.

As this drug has not been tried in tic douloureux, this study reports its effects on 25 such patients affected by 30 different attacks.

\section{METHOD}

PATIENTS The patients were between 30 and 77 years of age, with a mean of 58 years; 21 were older than 50 years. Nineteen were females.

The selection of patients was made mainly by their failure to respond to carbamazepine: 19 had been treated with this drug, with no response in 16. One had been helped by phenytoin several years 
previously, but did not respond in a recent recurrence. They had all tried multiple oral and parenteral analgesics without benefit.

All the patients fulfilled the clinical criteria for the diagnosis of the condition: (1) recurrent short paroxysms of severe pain localized to the cutaneous distribution of one or more branches of the trigeminal nerve; (2) absence of sensory loss or motor weakness in the peripheral distribution of the fifth nerve; (3) lack of neurovegetative signs (rhinorrhoea, epiphora, facial erythema), as a differential point from the so-called 'atypical facial neuralgias' such as sphenopalatine neuralgia; (4) normal radiographic studies of the skull and cranial cavities, as well as no history of progressive neurological dysfunction.

All the patients admitted to the study had 10 or more paroxysms of pain every day; a majority of them had as many as several per hour.

PROCEDURE The drug was given to every patient in slowly increasing doses in order to assure a good tolerance. The initial dose was $1.0 \mathrm{mg}$ twice or three times a day, progressing to a dose of 6 to $8 \mathrm{mg}$ over a period of approximately 10 days. In 19 patients $(76 \%)$ we could reach this recommended dose.

If the drug were unsuccesful after a trial of two to four weeks, it was discontinued and considered a failure. When the response was good, which usually happened after about 10 days of treatment, administration was maintained for a period of six to nine weeks.

The clinical evaluation of the effect of the drug was made every one to seven days, noting the variations in the frequency of the paroxysms, the subjective impression about its severity, and the intensity of the side-effects. The effectiveness of the drug was tabulated on three levels: (1) disappearance of the paroxysms; (2) reduction in their frequency by at least $80 \%$ (reduction from 10 crises or more per day to one or two as a maximum); (3) no effect on the frequency of the paroxysms, or reduction by less than $80 \%$.

\section{RESULTS}

In 30 episodes of tic douloureux treated with clonazepam in 25 patients, the pain disappeared in only $40 \%$, but an additional $23.3 \%$ were clearly helped by the drug. The failures accounted for the remaining one-third of the patients.

The most prominent side-effects were somnolence and unsteadiness of gait. In all except three patients, somnolence was present, and its intensity was proportional to the dose of clonazepam. In patients older than 60 years, hypersomnia was constant during day and night. The unsteadiness of gait was present in 20 patients and manifested itself by lateral deviations and occasional falls. In most patients unsteadiness of gait was slight and transient, but in those older than 60 it was so severe that they had to stay permanently in bed. This gait unsteadiness was unaccompanied by limb-ataxia, nystagmus, or dysarthria. Two patients (67 and 77 years old) showed a transient period of mental confusion which disappeared after discontinuing the drug.

\section{DISCUSSION}

The effectiveness of clonazepam in epilepsy has been demonstrated by several authors (Gastaut, 1970; Bonduelle and Sallou, 1973). In experimental conditions, it increases the electroconvulsive threshold in the cat and rat. It also has a weak relaxing effect on striated musculature which is five times less potent than with chlordiazepoxide.

Our results suggest that clonazepam is also effective in the control of the paroxysms of pain of tic douloureux. Although our study was not a double-blind trial, a placebo effect is unlikely for the following reasons: (1) all the patients had tried several analgesics and 16 of our group of 25 had used carbamazepine in the past, without control of the neuralgia; (2) only the cases with total disappearance or significant reduction (by $80-90 \%$ ) of the neuralgia were considered successful.

From our results, it can be concluded that clonazepam is a useful drug in the treatment of tic douloureux, with a clinical effectiveness in about $65 \%$ of the cases. This level of therapeutic success is comparable with phenytoin, which, in different series, has a clinical value in about $70 \%$ of cases (Braham and Saiz, 1960), with disappearance of the neuralgia in half of them (Blom, 1962). On the other hand, clonazepam is less effective than carbamazepine which has clinical value in $80-90 \%$ of the cases (Blom, 1962) and remains the drug of choice for this disease. However, 16 of our 25 patients had been previously resistant to this drug, and eight of them showed complete disappearance and one partial reduction of the neuralgia while on 
clonazepam; this suggests that clonazepam should be considered as a second choice after proven failure with carbamazepine.

The three drugs mentioned in this report are useful for the treatment of the crisis of tic douloureux, but they do not prevent the recurrence of new crises that will force a reinstitution of therapy. An important limiting factor for the clinical use of this drug is the frequency and severity of its side-effects, somnolence and unsteadiness of gait (present to some extent in 80 and $88 \%$ of the patients, respectively). However, they were severe and incapacitating in only nine patients, leaving a significant number $(64 \%)$ in which these side-effects were tolerable. On the other hand, the frequency and severity of the side-effects are probably related to the advanced age of the patients, because the use of this drug in a younger population of epileptics (Gastaut, 1970; Bonduelle and Sallou, 1973) in comparable or even higher doses, was followed by much less severe and only transient side-effects. Regarding other possible side-effects of clonazepam, experiences with more than 600 cases treated for epilepsy reported by the manufacturers, with serially controlled chemistry of blood, urine, and liver, no abnormalities were detected. For these reasons, we believe that, although this is a poorly tolerated drug in general, its use is clinically possible and is justified in young adults who have proven to be resistant to carbamazepine.

The action of phenytoin and carbamazepine in the control of tic douloureux is probably mediated by the blockage of synaptic transmission in polysynaptic circuits at the level of the trigeminal nuclei of the brain-stem (Fromm and Killian, 1967). This has been demonstrated in the intact anaesthetized cat with intracellular recordings of the spinal trigeminal nucleus, after peripheral stimulation of an afferent nerve (superior maxillary), before and after the intravenous administration of these drugs (Fromm and Landgren, 1963; Fromm and Killian, 1967). This effect was more prolonged and constant with carbamazepine than phenytoin, a fact that correlates with the better clinical results with the former drug.

Clonazepam is a proven anticonvulsant, as are phenytoin and carbamazepine. There are other anticonvulsants, such as phenobarbitone, which are not effective in trigeminal neuralgia, and where the mechanism of action in epilepsy probably relates to depression of axonal transmission and neuronal excitability (Fromm and Killian, 1967). From these data, we can postulate that clonazepam, an anticonvulsant and antineuralgic, possibly acts by the same mechanism of depression of central polysynaptic circuits which have been demonstrated for phenytoin and carbamazepine.

\section{REFERENCES}

Blom, S. (1962). Trigeminal neuralgia: its treatment with a new anticonvulsant drug (G-32883). Lancet, 1, 839-840.

Blom, S. (1963). Tic douloureux treated with a new anticonvulsant. Archives of Neurology (Chic.), 9, 285-290.

Bonduelle, M., and Sallou, C. (1973). Étude de 135 cas d'épilepsies traitées par le clonazepam. Semaine des Hôpitaux (Thérapeutique), 49, suppl. 10-20 July, 33-38.

Braham, J., and Saiz, A. (1960). Phenytoin in the treatment of trigeminal and other neuralgias. Lancet, 2, 892-893.

Fromm, G. H., and Killian, J. M. (1967). Effect of some anticonvulsant drugs on the spinal trigeminal nucleus. Neurology (Minneap.), 17, 275-280.

Fromm, G. H., and Landgren, S. (1963). Effect of diphenylhydantoin on single cells in the spinal trigeminal nucleus. Neurology (Minneap.), 13, 34-37.

Gastaut, H. (1970). Propriétés antiépileptiques exceptionelles d'une benzodiazépine nouvelle, le Ro 05-4023. La Vie Médicale, 51, 5175-5188. 